\title{
A case-control study of the association between the EGFR gene and glioma risk in a Chinese Han population
}

\author{
Mengdan Yan ${ }^{1,2}$, Jingjie $\mathrm{Li}^{1,2}$, Na He ${ }^{1,2}$, Xugang Shi ${ }^{2}$, Shuli Du ${ }^{2}$, Bin $\mathrm{Li}^{1,2,3}$ and Tianbo \\ Jin ${ }^{1,2}$ \\ ${ }^{1}$ Key Laboratory of Resource Biology and Biotechnology in Western China, Northwest University, Ministry of Education, Xi'an, \\ China \\ ${ }^{2}$ Xi'an Tiangen Precision Medical Research Institute, Xi'an, China \\ ${ }^{3}$ Department of Neurosurgery, Xi'an First Hospital, Xi'an, China \\ Correspondence to: Bin Li, email: libinwhu@nwu.edu.cn
}

Tianbo Jin, email: jintianbo@gmail.com

Keywords: glioma, EGFR gene, tag single nucleotide polymorphism, case-control study

Received: July 13,2016 Accepted: December 07, $2016 \quad$ Published: April 07, 2017

Copyright: Yan et al. This is an open-access article distributed under the terms of the Creative Commons Attribution License 3.0 (CC BY 3.0), which permits unrestricted use, distribution, and reproduction in any medium, provided the original author and source are credited.

\section{ABSTRACT}

The Epidermal Growth Factor Receptor gene has been reported to be involved in the progression of gliomas which is one of the deadliest primary brain tumors in humans. To determine potential association between EGFR and glioma risk, we performed a case-control study with 394 glioma patients and 298 cancer-free controls in which captured a total of 8 tag single nucleotide polymorphisms of EGFR gene from Xi'an, China. SPSS 19.0 statistical packages, $X^{2}$ test, genetic model analysis and SHEsis software platform were analyzed $s$ the variants in EGFR gene associations with glioma risk. For five different inheritance models analyzed, the following genotypes were associated with increased glioma risk. In the codominant model, genotype CC (rs730437, $O R=1.93, p=0.024 ; r s 1468727, O R=2.02, p=0.007)$; In the dominant model, genotype CA and CC $(r s 730437$, OR $=1.45, p=0.026)$, genotype GA and AA (rs845552, OR $=1.40, p=0.044)$; In the recessive model, genotype CC (rs730437, OR $=1.64, p=0.026 ;$ rs1468727, OR $=1.87, p=0.002) ;$ In the additive model, genotype CC $(r s 730437$, OR $=1.32, p=0.006 ;$ rs1468727, OR = 1.39, $p=0.005)$, genotype GG $(r s 11506105$, OR $=1.32, p=0.02)$ and genotype AA $(r s 845552, O R=1.27, p=$ 0.04). Our study indicated that 8 mutants located in EGFR gene were risk-conferring factors, larger and different populations with EGFR polymorphisms are required to verify these associations.

\section{INTRODUCTION}

Glioma is the most common primary central nervous system (CNS) tumors that originate from glia, accounting for approximately $80 \%$ of all primary malignant brain tumors, with nearly 20,000 people affected annually in the United States [1]. The survival time and prognosis of patients diagnosed with gliomas in adults is very poor. According to the WHO classification (2007), gliomas are classified into four grades (I to IV) based on microscopic appearance [2]. Alternatively, they are divided into astrocytic tumors, oligodendrogliomas, and oligoastrocytomas. Despite high-dosage ionizing irradiation (IR) devoted to the risk factor for gliomas, the etiology of sporadic gliomas remains unexplained [3]. In addition, only a small proportion of individuals who are exposed to IR environments will develop gliomas, suggesting hereditary factors contribute to susceptibility to glioma.

The Epidermal Growth Factor Receptor (EGFR) is a transmembrane tyrosine kinase in $7 \mathrm{p} 11.2$, It is one of the critical oncogenes for several cancers and variably expressed from embryogenesis to adulthood in the normal brain development. For low-grade glioma [4] and 
Table 1: General characteristics of glioma subjects and healthy controls

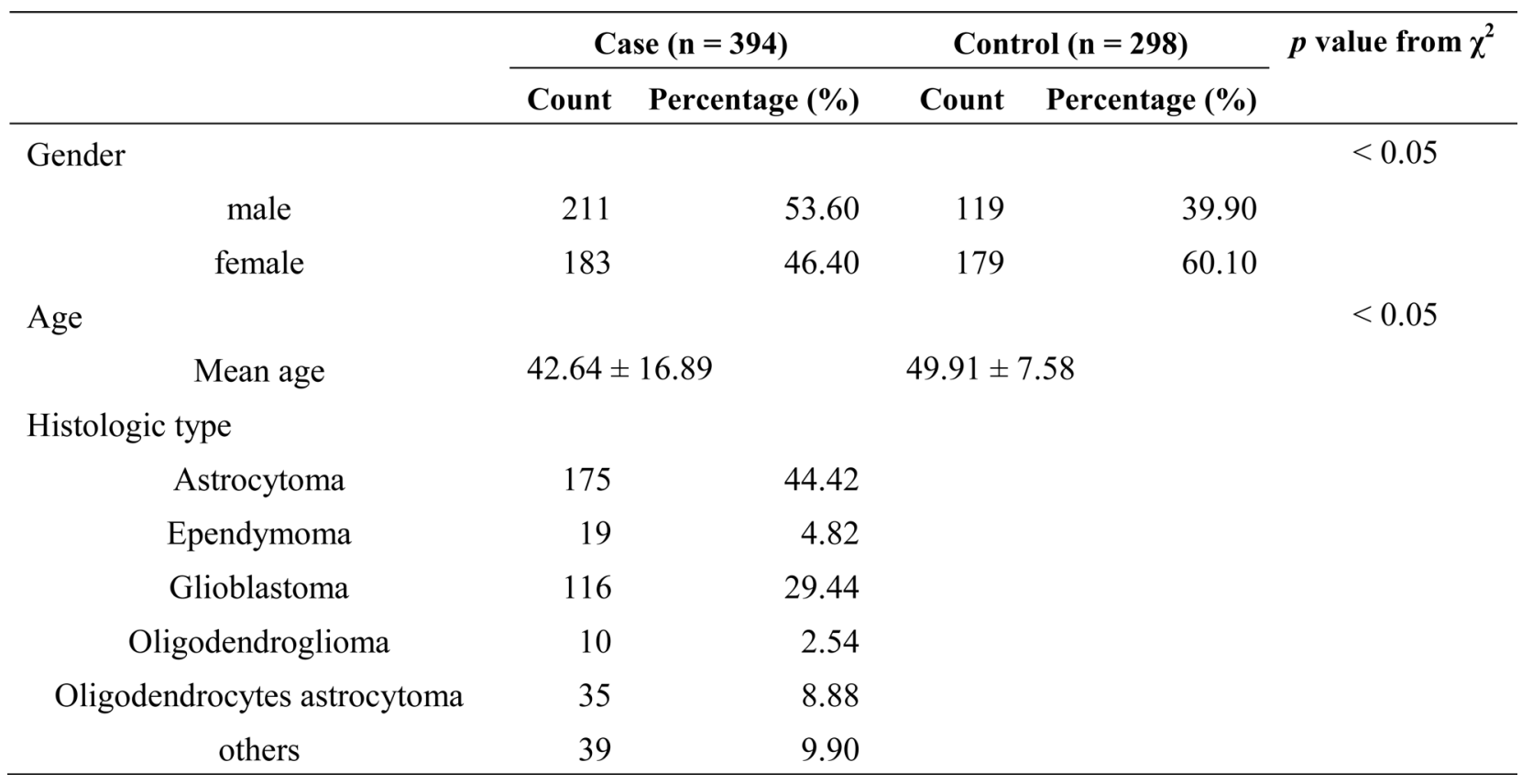

anaplastic astrocytoma [5], The overexpression of EGFR may be a prominent event in the progression of gliomas with a poor prognosis. Current evidence suggests that EGFR amplification is a hallmark of glioblastoma (GBM). As the most frequent and the most malignant glioma in the adult population [6-8], accounting for approximately $30 \%-50 \%$ of high-grade glioma and containing a genetic mutation of EGFR (EGFRvIII).

Although several studies showed that EGFR gene polymorphisms were associated with glioma risk, those currently available results are inconclusive [9-11]. The aim of this study was to investigate and validate the potential relationship between tSNPs in EGFR gene and glioma susceptibility in the Han Chinese population using a casecontrol study.

\section{RESULTS}

\section{Study participants}

The details of selected characteristics of all subjects were presented in Table 1, including gender, age, and histologic type. We enrolled a total of 692 participants, including 394 patients (211 males, 183 females) and 298 controls (119 males, 179 females) in this study, the mean age of the subjects was $42.64 \pm 16.89$ years for cases and $49.91 \pm 7.58$ years for controls. The distributions of gender and age were statistically significant differences between the case and control groups $(\mathrm{p}<0.05)$. Of the cancers, astrocytoma (175) and glioblastoma (116) are the more common histologies, and ependymoma (19), oligodendroglioma (10), oligodendrocytes astrocytoma
(35) and others (39) are the less common histologies.

\section{Hardy-Weinberg equilibrium and alleles of eight tSNPs}

A total of 8 tag single nucleotide polymorphisms (tSNPs) were successfully genotyped in all participants, and all of the tested tSNPs were in accordance with Hardy-Weinberg equilibrium (HWE) in control subjects $(p>0.05)$.We assumed that the minor allele of each tSNP was a risk factor compared to the wild-type allele. MAF in cases and controls were showed in Table 2. Using the $\chi^{2}$ test, three tSNPs were found to be associated with glioma and risk at a 5\% level (rs11506105, OR $=1.26$, $95 \% \mathrm{CI}=1.01-1.57, p=0.042 ;$ rs $1468727, \mathrm{OR}=1.31$, $95 \% \mathrm{CI}=1.05-1.62, p=0.016 ;$ rs $730437, \mathrm{OR}=1.33,95 \%$ $\mathrm{CI}=1.07-1.66, p=0.01)$. Given the number of glioma subjects, we only performed the stratified analysis with more common histologies (astrocytoma and glioblastoma), and also detected that three tSNPs were associated with an increased risk of astrocytoma ( $\mathrm{rs} 11506105, \mathrm{OR}=1.32$, $95 \% \mathrm{CI}=1.00-1.73, p=0.046 ; \mathrm{rs} 1468727, \mathrm{OR}=1.37$, $95 \% \mathrm{CI}=1.05-1.79, p=0.02 ; \mathrm{rs} 730437, \mathrm{OR}=1.42$, $95 \% \mathrm{CI}=1.09-1.86, p=0.01)$. However, there was no association of all tSNPs with risk of glioblastoma (Table $3)$.

\section{Association between EGFR and glioma risk}

We further analyzed the association between EGFR tSNPs and glioma risk under five gene models (codominant, dominant, recessive, overdominant and additive). Table 4 showed that the genotypes of tSNPs 
Table 2: Basic information about $E G F R$ candidate tSNPs in this study

\begin{tabular}{|c|c|c|c|c|c|c|c|c|c|c|c|}
\hline \multirow{2}{*}{ SNP ID } & \multirow{2}{*}{ Location } & \multirow{2}{*}{ Position } & \multirow{2}{*}{ Gene } & \multirow{2}{*}{ Role } & \multicolumn{2}{|c|}{$\begin{array}{c}\text { Glioma } \\
\text { frequency (MAF) }\end{array}$} & \multicolumn{2}{|c|}{$\begin{array}{c}\text { Glioblastoma } \\
\text { frequency (MAF) }\end{array}$} & \multicolumn{2}{|c|}{$\begin{array}{c}\text { Astrocytoma } \\
\text { frequency (MAF) }\end{array}$} & \multirow{2}{*}{$\begin{array}{c}\text { HWE } \\
p \text {-value }\end{array}$} \\
\hline & & & & & Case & Control & Case & Control & Case & Control & \\
\hline rs11506105 & $7 \mathrm{p} 11.2$ & 55220177 & $E G F R$ & Intron (boundary) & 0.41 & 0.356 & 0.414 & 0.586 & 0.171 & 0.829 & 0.898 \\
\hline rs12718945 & $7 \mathrm{p} 11.2$ & 55192963 & $E G F R$ & Intron & 0.354 & 0.343 & 0.341 & 0.659 & 0.109 & 0.891 & 0.898 \\
\hline rs 1468727 & $7 \mathrm{p} 11.2$ & 55230105 & $E G F R$ & Intron & 0.496 & 0.43 & 0.478 & 0.522 & 0.169 & 0.831 & 0.278 \\
\hline rs17172432 & $7 \mathrm{p} 11.2$ & 55141317 & $E G F R$ & Intron & 0.096 & 0.108 & 0.099 & 0.901 & 0.100 & 0.900 & 0.760 \\
\hline rs3752651 & $7 \mathrm{p} 11.2$ & 55229543 & $E G F R$ & Intron & 0.083 & 0.074 & 0.073 & 0.927 & 0.355 & 0.645 & 1.000 \\
\hline rs4947492 & $7 \mathrm{p} 11.2$ & 55187992 & $E G F R$ & Intron & 0.359 & 0.347 & 0.345 & 0.655 & 0.422 & 0.578 & 0.899 \\
\hline rs 730437 & $7 \mathrm{p} 11.2$ & 55215018 & $E G F R$ & Intron & 0.433 & 0.364 & 0.431 & 0.569 & 0.509 & 0.491 & 1.000 \\
\hline rs 845552 & $7 \mathrm{p} 11.2$ & 55245507 & $E G F R$ & Intron & 0.411 & 0.361 & 0.409 & 0.591 & 0.417 & 0.583 & 0.370 \\
\hline
\end{tabular}

Abbreviation: MAF: minor allele frequency

Table 3: Association between tSNPs in EGFR gene and risk of different histological types of gliomas in allelic model analysis

\begin{tabular}{|c|c|c|c|c|c|c|c|c|c|c|}
\hline \multirow{2}{*}{ SNP ID } & \multirow{2}{*}{$\begin{array}{c}\text { Base } \\
\text { change }\end{array}$} & \multicolumn{3}{|c|}{ Glioma } & \multicolumn{3}{|c|}{ Astrocytoma } & \multicolumn{3}{|c|}{ Glioblastoma } \\
\hline & & ORs $(95 \% \mathrm{CI})^{\mathrm{c}}$ & $p$-value & $\begin{array}{l}p \text { value } \\
\text { adj. * }\end{array}$ & ORs $(95 \% C I)$ & $p$-value & $\begin{array}{l}p \text { value } \\
\text { adj. * }\end{array}$ & ORs $(95 \% \mathrm{CI})$ & $p$-value & $\begin{array}{l}p \text { value } \\
\text { adj. * }\end{array}$ \\
\hline rs11506105 & $\mathrm{A} / \mathrm{G}$ & $1.26(1.01-1.57)$ & $0.042 *$ & 0.336 & $1.32(1.00-1.73)$ & $0.046^{*}$ & 0.368 & $1.28(0.93-1.74)$ & 0.125 & 1 \\
\hline rs 12718945 & $\mathrm{G} / \mathrm{T}$ & $1.05(0.84-1.32)$ & 0.663 & 1 & $1.06(0.80-1.40)$ & 0.696 & 1 & $0.99(0.72-1.36)$ & 0.948 & 1 \\
\hline rs 1468727 & $\mathrm{~T} / \mathrm{C}$ & $1.31(1.05-1.62)$ & $0.016^{*}$ & 0.128 & $1.37(1.05-1.79)$ & $0.020^{*}$ & 0.160 & $1.21(0.89-1.65)$ & 0.217 & 1 \\
\hline rs17172432 & $\mathrm{T} / \mathrm{C}$ & $0.88(0.62-1.25)$ & 0.478 & 1 & $0.92(0.59-1.42)$ & 0.695 & 1 & $0.91(0.55-1.50)$ & 0.706 & 1 \\
\hline rs3752651 & $\mathrm{T} / \mathrm{C}$ & $1.13(0.76-1.68)$ & 0.556 & 1 & $1.14(0.70-1.85)$ & 0.608 & 1 & $0.99(0.55-1.77)$ & 0.969 & 1 \\
\hline rs4947492 & $\mathrm{A} / \mathrm{G}$ & $1.06(0.84-1.32)$ & 0.635 & 1 & $1.06(0.80-1.40)$ & 0.682 & 1 & $0.99(0.72-1.36)$ & 0.957 & 1 \\
\hline rs730437 & $\mathrm{A} / \mathrm{C}$ & $1.33(1.07-1.66)$ & $0.010^{*}$ & 0.080 & $1.42(1.09-1.86)$ & $0.010^{*}$ & 0.080 & $1.32(0.97-1.80)$ & 0.075 & 0.600 \\
\hline rs845552 & $\mathrm{G} / \mathrm{A}$ & $1.24(0.99-1.54)$ & 0.062 & 1 & $1.27(0.97-1.67)$ & 0.086 & 0.688 & $1.23(0.90-1.68)$ & 0.195 & 1 \\
\hline
\end{tabular}

Abbreviations: OR: odds ratio; 95\% $\mathrm{CI}$ : 95\% confidence interval.

$* \mathrm{p} \leq 0.05$ indicates statistical significance.

$*$ p value was adjusted by Bonferroni corrections.

were associated with increased glioma risk: in the codominant model, genotype CC (rs730437, OR $=1.93$, 95\%CI: $1.19-3.13, p=0.024 ; \mathrm{rs} 1468727, \mathrm{OR}=2.02$, 95\% $\mathrm{CI}: 1.26-3.24, \mathrm{p}=0.007)$; In the dominant model, genotype $\mathrm{CA}$ and $\mathrm{CC}$ (rs730437, OR $=1.45,95 \% \mathrm{CI}$ : $1.05-2.02, p=0.026$ ), genotype GA and AA (rs845552, $\mathrm{OR}=1.40,95 \% \mathrm{CI}: 1.01-1.94, p=0.044)$; In the recessive model, genotype CC (rs730437, OR $=1.64,95 \% \mathrm{CI}$ : 1.06 $2.56, p=0.026 ; \mathrm{rs} 1468727, \mathrm{OR}=1.87,95 \% \mathrm{CI}: 1.25-2.81$, $p=0.002)$; In the additive model, genotype CC (rs730437, $\mathrm{OR}=1.37,95 \% \mathrm{CI}: 1.09-1.73, p=0.006 ; \mathrm{rs} 1468727$, $\mathrm{OR}=1.39,95 \% \mathrm{CI}: 1.10-1.75, p=0.005)$, genotype GG (rs11506105, OR $=1.32,95 \% \mathrm{CI}: 1.04-1.67, p=0.02)$ and genotype AA (rs845552, OR $=1.27,95 \% \mathrm{CI}$ : 1.01-1.59, $p$ $=0.04$ ). In addition, we observed the association between the EGFR and increased astrocytoma risk (Table 5), in the codominant model, genotype CC (rs730437, OR $=2.27$, 95\%CI: $1.26-4.10, p=0.024$; rs 1468727 , OR $=2.09$, 95\% $\%$ : $1.17-3.72, p=0.022)$; In the recessive model, genotype $\mathrm{CC}(\mathrm{rs} 730437, \mathrm{OR}=2.00,95 \% \mathrm{CI}: 1.17-3.41$, $p=0.011 ; \mathrm{rs} 1468727, \mathrm{OR}=1.99,95 \% \mathrm{CI}: 1.22-3.24, p$
$=0.0062)$ and genotype $\mathrm{GG}(\mathrm{rs} 11506105, \mathrm{OR}=1.86$, 95\%CI: $1.07-3.23, p=0.029)$. In the additive model, genotype CC (rs730437, OR $=1.46,95 \% \mathrm{CI}: 1.10-1.95$, $p=0.0095 ;$ rs $1468727, \mathrm{OR}=1.42,95 \% \mathrm{CI}: 1.06-1.90, p$ $=0.018)$, genotype $\mathrm{GG}(\mathrm{rs} 11506105, \mathrm{OR}=1.34,95 \% \mathrm{CI}$ : $1.01-1.80, p=0.045)$.

\section{Haplotype analysis}

Haplotype analysis detected the existence of two blocks in EGFR tSNPs (Figure 1), LD was found between rs4947492 and rs12718945, among rs730437, rs11506105 and rs3752651. Besides, we found that the CGT haplotype was associated with increased glioma risk $(\mathrm{OR}=1.41$, 95\%CI: 1.11-1.79, $p=0.006)$, CGT haplotype and CAT haplotype with an increased risk of astrocytoma (OR = 1.45, 95\%CI: $1.07-1.96, p=0.016$; OR $=3.11,95 \% \mathrm{CI}$ : $1.06-9.10, p=0.039$ ) (Table 6). 
Table 4: Relationship between $E G F R$ tSNPs and glioma cancer risk under multiple models of inheritance

\begin{tabular}{|c|c|c|c|c|c|c|c|c|}
\hline \multirow{2}{*}{ SNP ID } & \multirow{2}{*}{ Model } & \multirow{2}{*}{ Genotype } & \multirow{2}{*}{ control } & \multirow{2}{*}{ case } & \multicolumn{2}{|c|}{ crude analysis } & \multicolumn{2}{|c|}{ adjusted by age and gender } \\
\hline & & & & & OR $(95 \% \mathrm{CI})$ & $p$-value & OR $(95 \% \mathrm{CI})$ & $p$-value \\
\hline \multirow{9}{*}{ rs730437 } & \multirow[t]{3}{*}{ Codominant } & $\mathrm{A} / \mathrm{A}$ & $120(40.3 \%)$ & $126(32 \%)$ & 1 & \multirow[t]{3}{*}{0.035} & 1 & \multirow[t]{3}{*}{$0.024 *$} \\
\hline & & $\mathrm{C} / \mathrm{A}$ & $139(46.6 \%)$ & $195(49.5 \%)$ & $1.34(0.96-1.86)$ & & $1.33(0.94-1.87)$ & \\
\hline & & $\mathrm{C} / \mathrm{C}$ & $39(13.1 \%)$ & $73(18.5 \%)$ & $1.78(1.12-2.83)$ & & $1.93(1.19-3.13)$ & \\
\hline & \multirow[t]{2}{*}{ Dominant } & $\mathrm{A} / \mathrm{A}$ & $120(40.3 \%)$ & $126(32 \%)$ & 1 & \multirow[t]{2}{*}{0.024} & 1 & \multirow[t]{2}{*}{$0.026^{*}$} \\
\hline & & $\mathrm{C} / \mathrm{A}-\mathrm{C} / \mathrm{C}$ & $178(59.7 \%)$ & $268(68 \%)$ & $1.43(1.05-1.96)$ & & $1.45(1.05-2.02)$ & \\
\hline & \multirow[t]{2}{*}{ Recessive } & $\mathrm{A} / \mathrm{A}-\mathrm{C} / \mathrm{A}$ & $259(86.9 \%)$ & $321(81.5 \%)$ & 1 & \multirow[t]{2}{*}{0.052} & 1 & \multirow[t]{2}{*}{$0.026^{*}$} \\
\hline & & $\mathrm{C} / \mathrm{C}$ & $39(13.1 \%)$ & $73(18.5 \%)$ & $1.51(0.99-2.30)$ & & $1.64(1.06-2.56)$ & \\
\hline & \multirow{2}{*}{ Overdominant } & $\mathrm{A} / \mathrm{A}-\mathrm{C} / \mathrm{C}$ & $159(53.4 \%)$ & $199(50.5 \%)$ & 1 & \multirow[t]{2}{*}{0.460} & 1 & \multirow[t]{2}{*}{0.600} \\
\hline & & $\mathrm{C} / \mathrm{A}$ & $139(46.6 \%)$ & $195(49.5 \%)$ & $1.12(0.83-1.52)$ & & $1.09(0.79-1.49)$ & \\
\hline \multirow{11}{*}{ rs11506105 } & Log-additive & --- & -- & --- & $1.34(1.07-1.66)$ & 0.010 & $1.37(1.09-1.73)$ & $0.006^{*}$ \\
\hline & \multirow[t]{3}{*}{ Codominant } & $\mathrm{A} / \mathrm{A}$ & $120(41.1 \%)$ & $134(34.4 \%)$ & 1 & \multirow[t]{3}{*}{0.120} & 1 & \multirow[t]{3}{*}{0.064} \\
\hline & & $\mathrm{A} / \mathrm{G}$ & $136(46.6 \%)$ & $192(49.2 \%)$ & $1.26(0.91-1.76)$ & & $1.28(0.91-1.81)$ & \\
\hline & & $\mathrm{G} / \mathrm{G}$ & $36(12.3 \%)$ & $64(16.4 \%)$ & $1.59(0.99-2.56)$ & & $1.78(1.08-2.93)$ & \\
\hline & Dominant & $\mathrm{A} / \mathrm{A}$ & $120(41.1 \%)$ & $134(34.4 \%)$ & 1 & 0.072 & 1 & 0.053 \\
\hline & & A/G-G/G & $172(58.9 \%)$ & $256(65.6 \%)$ & $1.33(0.97-1.82)$ & & $1.38(1.00-1.92)$ & \\
\hline & Recessive & $\mathrm{A} / \mathrm{A}-\mathrm{A} / \mathrm{G}$ & $256(87.7 \%)$ & $326(83.6 \%)$ & 1 & 0.130 & 1 & 0.061 \\
\hline & & $\mathrm{G} / \mathrm{G}$ & $36(12.3 \%)$ & $64(16.4 \%)$ & $1.40(0.90-2.17)$ & & $1.55(0.97-2.45)$ & \\
\hline & Overdominant & $\mathrm{A} / \mathrm{A}-\mathrm{G} / \mathrm{G}$ & $156(53.4 \%)$ & $198(50.8 \%)$ & 1 & 0.490 & 1 & 0.570 \\
\hline & & $\mathrm{A} / \mathrm{G}$ & $136(46.6 \%)$ & $192(49.2 \%)$ & $1.11(0.82-1.51)$ & & $1.10(0.80-1.51)$ & \\
\hline & Log-additive & --- & -- & -- & $1.26(1.01-1.58)$ & 0.041 & $1.32(1.04-1.67)$ & $0.020 *$ \\
\hline & Codominant & $\mathrm{T} / \mathrm{T}$ & $88(30.8 \%)$ & $102(26.1 \%)$ & 1 & 0.024 & 1 & $0.007^{*}$ \\
\hline & & $\mathrm{T} / \mathrm{C}$ & $150(52.5 \%)$ & $190(48.6 \%)$ & $1.09(0.77-1.56)$ & & $1.13(0.77-1.64)$ & \\
\hline & & $\mathrm{C} / \mathrm{C}$ & $48(16.8 \%)$ & $99(25.3 \%)$ & $1.78(1.14-2.78)$ & & $2.02(1.26-3.24)$ & \\
\hline & Dominant & $\mathrm{T} / \mathrm{T}$ & $88(30.8 \%)$ & $102(26.1 \%)$ & 1 & 0.180 & 1 & 0.110 \\
\hline 1468777 & & $\mathrm{~T} / \mathrm{C}-\mathrm{C} / \mathrm{C}$ & $198(69.2 \%)$ & $289(73.9 \%)$ & $1.26(0.90-1.77)$ & & $1.33(0.93-1.90)$ & \\
\hline rs $1408 / 27$ & Recessive & $\mathrm{T} / \mathrm{T}-\mathrm{T} / \mathrm{C}$ & $238(83.2 \%)$ & $292(74.7 \%)$ & 1 & 0.007 & 1 & $0.002 *$ \\
\hline & & $\mathrm{C} / \mathrm{C}$ & $48(16.8 \%)$ & $99(25.3 \%)$ & $1.68(1.14-2.47)$ & & $1.87(1.25-2.81)$ & \\
\hline & Overdominant & $\mathrm{T} / \mathrm{T}-\mathrm{C} / \mathrm{C}$ & $136(47.5 \%)$ & $201(51.4 \%)$ & 1 & 0.320 & 1 & 0.280 \\
\hline & & $\mathrm{T} / \mathrm{C}$ & $150(52.5 \%)$ & $190(48.6 \%)$ & $0.86(0.63-1.16)$ & & $0.84(0.61-1.15)$ & \\
\hline & Log-additive & --- & --- & --- & $1.31(1.05-1.63)$ & 0.015 & $1.39(1.10-1.75)$ & $0.005^{*}$ \\
\hline & Codominant & $\mathrm{G} / \mathrm{G}$ & $121(42.2 \%)$ & $140(35.7 \%)$ & 1 & 0.180 & 1 & 0.110 \\
\hline & & G/A & $125(43.5 \%)$ & $182(46.4 \%)$ & $1.26(0.90-1.76)$ & & $1.35(0.95-1.92)$ & \\
\hline & & $\mathrm{A} / \mathrm{A}$ & $41(14.3 \%)$ & $70(17.9 \%)$ & $1.48(0.94-2.33)$ & & $1.55(0.96-2.51)$ & \\
\hline & Dominant & $\mathrm{G} / \mathrm{G}$ & $121(42.2 \%)$ & $140(35.7 \%)$ & 1 & 0.088 & 1 & $0.044 *$ \\
\hline 845552 & & $\mathrm{G} / \mathrm{A}-\mathrm{A} / \mathrm{A}$ & $166(57.8 \%)$ & $252(64.3 \%)$ & $1.31(0.96-1.79)$ & & $1.40(1.01-1.94)$ & \\
\hline rs845552 & Recessive & G/G-G/A & $246(85.7 \%)$ & $322(82.1 \%)$ & 1 & 0.210 & 1 & 0.210 \\
\hline & & $\mathrm{A} / \mathrm{A}$ & $41(14.3 \%)$ & $70(17.9 \%)$ & $1.30(0.86-1.98)$ & & $1.32(0.85-2.05)$ & \\
\hline & Overdominant & G/G-A/A & $162(56.5 \%)$ & $210(53.6 \%)$ & 1 & 0.460 & 1 & 0.300 \\
\hline & & G/A & $125(43.5 \%)$ & $182(46.4 \%)$ & $1.12(0.83-1.53)$ & & $1.19(0.86-1.64)$ & \\
\hline & Log-additive & --- & --- & --- & $1.22(0.98-1.52)$ & 0.067 & $1.27(1.01-1.59)$ & $0.040^{*}$ \\
\hline
\end{tabular}

Abbreviations: OR: odds ratio; 95\%CI: 95\% confidence interval.

$* p \leq 0.05$ indicates statistical significance.

\section{DISCUSSION}

We successfully genotyped 8 tSNPs in EGFR and found some evidence of association at 3 SNPs (rs1468727, rs 730437, and rs11506105) that were associated with glioma risk. In addition, CGT and CAT in block 3 trends to increase glioma risk, which suggested that this gene may contribute to glioma in a case-control study from the Han Chinese population. EGFR (erbB-1), the prototypical member of the erbB receptor family and is frequently expressed in human tissues. The EGFR signaling pathway regulates a wide range of cellular activities associated with cell growth, migration and survival [12]. Several previous studies have assessed single nucleotide polymorphisms 
Table 5: Association between $E G F R$ tSNPs and risk of astrocytoma under multiple models of inheritance

\begin{tabular}{|c|c|c|c|c|c|c|c|c|}
\hline \multirow{2}{*}{ SNP ID } & \multirow{2}{*}{ Model } & \multirow{2}{*}{ Genotype } & \multirow{2}{*}{ control } & \multirow{2}{*}{ case } & \multicolumn{2}{|c|}{ crude analysis } & \multicolumn{2}{|c|}{ adjusted by age and gender } \\
\hline & & & & & OR $(95 \% \mathrm{CI})$ & $p$-value & OR $(95 \%$ CI) & $p$-value \\
\hline \multirow{12}{*}{ rs 730437} & \multirow[t]{3}{*}{ Codominant } & $\mathrm{A} / \mathrm{A}$ & $120(40.3 \%)$ & $54(30.9 \%)$ & 1 & 0.037 & 1 & $0.024 *$ \\
\hline & & $\mathrm{C} / \mathrm{A}$ & $139(46.6 \%)$ & $85(48.6 \%)$ & $1.36(0.89-2.07)$ & & $1.26(0.81-1.96)$ & \\
\hline & & $\mathrm{C} / \mathrm{C}$ & $39(13.1 \%)$ & $36(20.6 \%)$ & $2.05(1.18-3.57)$ & & $2.27(1.26-4.10)$ & \\
\hline & \multirow[t]{2}{*}{ Dominant } & $\mathrm{A} / \mathrm{A}$ & $120(40.3 \%)$ & $54(30.9 \%)$ & 1 & 0.039 & 1 & $0.072 *$ \\
\hline & & $\mathrm{C} / \mathrm{A}-\mathrm{C} / \mathrm{C}$ & $178(59.7 \%)$ & $121(69.1 \%)$ & $1.51(1.02-2.24)$ & & $1.46(0.96-2.22)$ & \\
\hline & \multirow[t]{2}{*}{ Recessive } & $\mathrm{A} / \mathrm{A}-\mathrm{C} / \mathrm{A}$ & $259(86.9 \%)$ & $139(79.4 \%)$ & 1 & 0.034 & 1 & $0.011 *$ \\
\hline & & $\mathrm{C} / \mathrm{C}$ & $39(13.1 \%)$ & $36(20.6 \%)$ & $1.72(1.05-2.83)$ & & $2.00(1.17-3.41)$ & \\
\hline & \multirow[t]{2}{*}{ Overdominant } & $\mathrm{A} / \mathrm{A}-\mathrm{C} / \mathrm{C}$ & $159(53.4 \%)$ & $90(51.4 \%)$ & 1 & 0.69 & 1 & 0.89 \\
\hline & & $\mathrm{C} / \mathrm{A}$ & $139(46.6 \%)$ & $85(48.6 \%)$ & $1.08(0.74-1.57)$ & & $0.97(0.65-1.45)$ & \\
\hline & Log-additive & --- & --- & --- & $1.42(1.08-1.86)$ & 0.011 & $1.46(1.10-1.95)$ & $0.0095 *$ \\
\hline & Codominant & $\mathrm{A} / \mathrm{A}$ & $120(41.1 \%)$ & $59(34.1 \%)$ & 1 & 0.12 & 1 & $0.079 *$ \\
\hline & & $\mathrm{A} / \mathrm{G}$ & $136(46.6 \%)$ & $82(47.4 \%)$ & $1.23(0.81-1.86)$ & & $1.13(0.73-1.76)$ & \\
\hline \multirow{7}{*}{ rs11506105 } & & $\mathrm{G} / \mathrm{G}$ & $36(12.3 \%)$ & $32(18.5 \%)$ & $1.81(1.02-3.19)$ & & $1.99(1.09-3.63)$ & \\
\hline & Dominant & $\mathrm{A} / \mathrm{A}$ & $120(41.1 \%)$ & $59(34.1 \%)$ & 1 & 0.13 & 1 & 0.21 \\
\hline & \multirow{3}{*}{ Recessive } & A/G-G/G & $172(58.9 \%)$ & $114(65.9 \%)$ & 1.35 (0.91-1.99) & & $1.30(0.86-1.96)$ & \\
\hline & & $\mathrm{A} / \mathrm{A}-\mathrm{A} / \mathrm{G}$ & $256(87.7 \%)$ & $141(81.5 \%)$ & 1 & 0.072 & 1 & $0.029 *$ \\
\hline & & $\mathrm{G} / \mathrm{G}$ & $36(12.3 \%)$ & $32(18.5 \%)$ & $1.61(0.96-2.71)$ & & $1.86(1.07-3.23)$ & \\
\hline & \multirow[t]{2}{*}{ Overdominant } & $\mathrm{A} / \mathrm{A}-\mathrm{G} / \mathrm{G}$ & $156(53.4 \%)$ & $91(52.6 \%)$ & 1 & 0.86 & 1 & 0.74 \\
\hline & & $\mathrm{A} / \mathrm{G}$ & $136(46.6 \%)$ & $82(47.4 \%)$ & $1.03(0.71-1.51)$ & & $0.93(0.63-1.39)$ & \\
\hline \multirow{11}{*}{ rs 1468727} & Log-additive & --- & -- & -- & $1.32(1.00-1.73)$ & 0.047 & $1.34(1.01-1.80)$ & $0.045^{*}$ \\
\hline & \multirow[t]{3}{*}{ Codominant } & $\mathrm{T} / \mathrm{T}$ & $88(30.8 \%)$ & $43(24.6 \%)$ & 1 & 0.041 & 1 & $0.022 *$ \\
\hline & & $\mathrm{T} / \mathrm{C}$ & $150(52.5 \%)$ & $86(49.1 \%)$ & $1.17(0.75-1.84)$ & & $1.08(0.67-1.74)$ & \\
\hline & & $\mathrm{C} / \mathrm{C}$ & $48(16.8 \%)$ & $46(26.3 \%)$ & $1.96(1.14-3.38)$ & & $2.09(1.17-3.72)$ & \\
\hline & \multirow[t]{2}{*}{ Dominant } & $\mathrm{T} / \mathrm{T}$ & $88(30.8 \%)$ & $43(24.6 \%)$ & 1 & 0.15 & 1 & 0.24 \\
\hline & & $\mathrm{T} / \mathrm{C}-\mathrm{C} / \mathrm{C}$ & $198(69.2 \%)$ & $132(75.4 \%)$ & $1.36(0.89-2.09)$ & & $1.31(0.84-2.05)$ & \\
\hline & \multirow[t]{2}{*}{ Recessive } & $\mathrm{T} / \mathrm{T}-\mathrm{T} / \mathrm{C}$ & $238(83.2 \%)$ & $129(73.7 \%)$ & 1 & 0.015 & 1 & $0.0062^{*}$ \\
\hline & & $\mathrm{C} / \mathrm{C}$ & $48(16.8 \%)$ & $46(26.3 \%)$ & $1.77(1.12-2.79)$ & & $1.99(1.22-3.24)$ & \\
\hline & \multirow[t]{2}{*}{ Overdominant } & $\mathrm{T} / \mathrm{T}-\mathrm{C} / \mathrm{C}$ & $136(47.5 \%)$ & $89(50.9 \%)$ & 1 & 0.49 & 1 & 0.25 \\
\hline & & $\mathrm{T} / \mathrm{C}$ & $150(52.5 \%)$ & $86(49.1 \%)$ & $0.88(0.60-1.28)$ & & $0.79(0.53-1.18)$ & \\
\hline & Log-additive & --- & --- & --- & $1.39(1.06-1.83)$ & 0.018 & $1.42(1.06-1.90)$ & $0.018 *$ \\
\hline
\end{tabular}

Abbreviations: OR: odds ratio; 95\%CI: 95\% confidence interval.

$* p \leq 0.05$ indicates statistical significance.

(SNPs) in the EGFR gene that is implicated in human tumors, such as breast cancer [13], lung cancer [14] and prostate cancer [15]. And several studies have demonstrated that certain genotypes of the EGFR gene may be related to glioma susceptibility, which indicated $E G F R$ polymorphisms play an important role in the carcinogensis of glioma $[11,16]$. Our results found that the $C$ allele of rs1468727 and rs730437, intronic SNPs within the EGFR gene, which was associated with an increased risk of glioma compared to controls. Previously, in Chinese populations, Liu et al suggested that the $\mathrm{T}$ allele of rs1468727 and C allele of rs730437 were associated with decreased glioma risk [10]; Wang et al observed that the $\mathrm{C}$ allele of rs 1468727 may increase the risk of glioma, while the A allele of rs730437 may decreased the risk of glioma [11], such inconsistencies in these reports may result from different environment or insufficient sample size. From one study of Andersson U et.al, we found the significant difference exist in these SNPs of our study.
For example rs3752651 which significant association with glioma in heterozygote genotype model $(\mathrm{OR}=1.29$, $95 \% \mathrm{CI}=1.06-1.56, p=0.008)[16]$, but in our study it isn't significant $(\mathrm{OR}=1.13,95 \% \mathrm{CI}=0.76-1.68, p=0.556)$. A study in Han Chinese population indicated that genotype CC of rs 1468727 and rs730437 conferred an elevated risk for glioma [9], which was well in accordance with our findings. All these papers examine implied that two single nucleotide polymorphisms (rs1468727 and rs730437) may play critical roles in the pathogenesis of glioma and might be potential molecular markers for evaluating glioma risk.

The SNP 11506105 in EGFR was identified to be associated with an increased risk of glioma, according to allele $\mathrm{G}$ association analysis, which is inconsistent with a previous study in a European population, the A allele of rs11506105 had been found to be associated with increased risk of glioma $(\mathrm{OR}=1.39,95 \% \mathrm{CI}$ : 1.08-1.78, $p$ $=0.012$ ) [16]. Our data for rs845552, we did not find any statistical association with astrocytoma risk, suggesting 
Table 6: Haplotype frequencies of $E G F R$ gene and association with risk of glioma and astrocytoma

\begin{tabular}{|c|c|c|c|c|c|c|c|c|}
\hline \multirow[b]{2}{*}{ rs 730437} & \multirow[b]{2}{*}{ rs11506105 } & \multirow[b]{2}{*}{ rs3752651 } & \multicolumn{3}{|c|}{ Glioma } & \multicolumn{3}{|c|}{ Astrocytoma } \\
\hline & & & Freq & $\begin{array}{c}\text { OR } \\
(95 \% \mathrm{CI})\end{array}$ & $p$-value & Freq & $\begin{array}{c}\text { OR } \\
(95 \% \mathrm{CI})\end{array}$ & $p$-value \\
\hline $\mathrm{A}$ & $\mathrm{A}$ & $\mathrm{T}$ & 0.5152 & 1 & --- & 0.5245 & 1 & --- \\
\hline $\mathrm{C}$ & G & $\mathrm{T}$ & 0.3855 & $\begin{array}{c}1.41 \\
(1.11-1.79)\end{array}$ & $0.006^{*}$ & 0.3781 & $\begin{array}{c}1.45 \\
(1.07-1.96)\end{array}$ & $0.016^{*}$ \\
\hline A & A & $\mathrm{C}$ & 0.0787 & $\begin{array}{c}1.37 \\
(0.89-2.11)\end{array}$ & 0.16 & 0.0768 & $\begin{array}{c}1.34 \\
(0.77-2.33)\end{array}$ & 0.31 \\
\hline $\mathrm{C}$ & A & $\mathrm{T}$ & 0.0177 & $\begin{array}{c}1.84 \\
(0.72-4.73)\end{array}$ & 0.21 & 0.0173 & $\begin{array}{c}3.11 \\
(1.06-9.10)\end{array}$ & $0.039 *$ \\
\hline
\end{tabular}

Abbreviations: OR: odds ratio; 95\%CI: 95\% confidence interval.

$* p \leq 0.05$ indicates statistical significance.

that either there was no such effect or the small sample size after stratified analysis limited statistical power.

Besides the allelic model analysis, we further performed genotypic model analysis to investigate the role of EGFR variants on glioma risk. Our study found that rs730437 and rs1468727 were associated with increased glioma risk in co-dominant, recessive and logadditive models (Table 4). The two SNPs were found to be associated with glioma risk in the many studies. However, the past results were inconsistent. The inconsistency might be attributed to the different ethnicity in the populations studied, given that the minor allele frequency was different. In addition, after a strict Bonferroni correction analysis was applied, the significance level of the association between $E G F R$ tSNPs and risk of glioma was attenuated.

There are several inherent limitations that cannot be ignored. Firstly, the subgroup analysis for histology and/or grade, and gender-specific significant variants in glioma patients were not performed, due to the relatively small sample size. Secondly, we selected tSNPs with MAF higher than 5\% in the HapMap Chinese Han Beijing

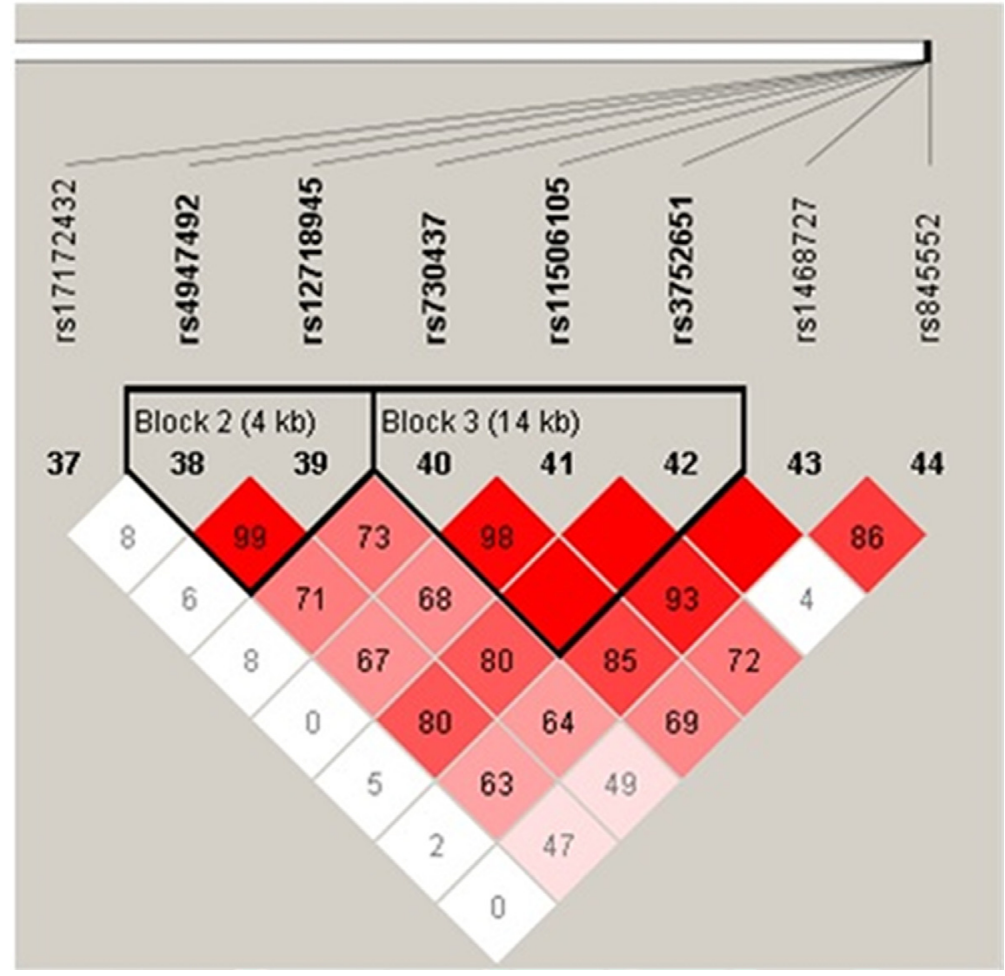

Figure 1: Haplotype block map for tSNPs in the EGFR gene. 
population to determine the statistical power was large enough for analyzing data, the haplotype-based study was also performed to affirm sufficiently high power to detect the association between candidate tSNPs and glioma risk. Thirdly, glioma patients and controls were drawn from same hospital, so selection bias cannot be excluded and the subjects might not be representative of the general population.

\section{CONCLUSIONS}

Our study is an exploratory research which shed new light on the association between EGFR tSNPs and glioma risk in the Chinese population. Combined with the previous studies, we only further ascertain the potential role of the EGFR gene to glioma onset. Further investigation with a larger sample size in Chinese populations or another ethnic is warranted.

\section{MATERIALS AND METHODS}

\section{Study population}

We recruited patients between December 2002 and April 2013 for the molecular epidemiology study at the First Affiliated Hospital of the Medical College of Xi'an Jiaotong University. All gliomas cases were confirmed by the newly diagnosed histopathologically, and patients who had any history of cancer and received either radiotherapy or chemotherapy before surgery were excluded. Through our normal the epidemiological investigation and eliminated individuals with any glioma disease family history of more than three generations, eventually a total of 298 controls during the similar period was randomly recruited from the medical examination center at First Affiliated Hospital. Their frequency matched to all adult brain tumor cases on age, sex, and region.These controls were cancer-free and genetically unrelated to the patients.

\section{Data collection}

Each participant was interviewed by a trained nurse, a standard questionnaire was used to collect detailed information regarding demographic characteristics, radiation exposure, smoking habit, alcohol drinking habit, family history of cancer and other factors. Each subject donated 3-5 $\mathrm{ml}$ of venous blood, and all subjects who agreed to the purpose and experimental procedures of the study, and signed their written informed consents prior to sample donation. The research protocol was performed in accordance with the Declaration of Helsinki and approved by the Human Research Committee of the First Affiliated Hospital of the Medical College of Xi' an Jiaotong University for Approval of Research Involving
Human Subjects.

\section{SNP selection and genotyping}

Using the public dbSNP (https://www.ncbi.nlm. nih.gov/snp) and HapMap (http://www.hapmap.org/) and identify linkage disequilibrium (LD) blocks within $E G F R$ to get a dense tagging of SNPs, we used Haploview software (http://www.broad.mit.edu/mpg/haploview/) setting the minimum r2 to 0.9 and the minimum minor allele frequency to $5 \%$ in HapMap Chinese Han Beijing residents. Finally we selected candidate 8 tSNPs in the $E G F R$ gene for genotyping, which previously reported being associated with gliomas [16]. Genomic DNA was extracted from whole blood using the GoldMag ${ }^{\circledR}$ nanoparticles method (GoldMag Ltd. Xi'an, China) according to the manufacturer's instructions, and the DNA concentration was measured with the NanoDrop 2000C (Thermo Scientific, Waltham, Massachusetts, USA). The Sequenom MassARRAY Assay Design 3.0 Software (San Diego, California, USA) was used to design Multiplexed SNP MassEXTEND assays [17]. SNPs genotyping was performed by the Sequenom MassARRAY RS1000 (San Diego, California, USA) and Sequenom Typer 4.0 Software (San Diego, California, USA) was used to perform data management and analysis as previously described $[17,18]$.

\section{Data analysis}

Statistical calculations were made using Microsoft Excel (Redmond, WA, USA) and SPSS 19.0 statistical package (SPSS, Chicago, IL, USA). All statistical tests were two-sided, and $p \leq 0.05$ was regarded as statistically significant differences. Each tSNP frequency was assessed for departure from Hardy-Weinberg Equilibrium (HWE) using an exact test among controls. We compared genotype frequencies and allele frequencies between cases and controls using the $\chi^{2}$ test. To estimate the association between the EGFR genetic polymorphisms and risk of glioma, as measured by odds ratios (ORs) and $95 \%$ confidence intervals (CIs) were calculated using unconditional logistic regression with adjustment for age and gender [19]. We used multiple inheritance models (codominant, dominant, recessive, overdominant and additive) to evaluate the associations between certain tSNPs in EGFR gene and glioma risk, with SNPstats software (http://bioinfo.iconcologia.net/snpstats/start.htm) [20]. For each polymorphism, ORs and $95 \%$ CIs were calculated by unconditional logistic regression analysis with and without adjustment of age and gender [19].

Finally, we used Haploview software package (version 4.2) to evaluate the pairwise linkage disequilibrium (LD), haplotype construction, and genetic association at polymorphism loci $[21,22]$. 


\section{CONFLICTS OF INTEREST}

The authors declare no competing financial interests.

\section{GRANT SUPPORT}

This work was supported by the Science Foundation of Northwest University (No. 15NW19) and the Science Foundation of Northwest University (No. 338020007).

\section{REFERENCES}

1. Dolecek TA, Propp JM, Stroup NE, Kruchko C. CBTRUS statistical report: primary brain and central nervous system tumors diagnosed in the United States in 2005-2009. Neurooncol. 2012; 14:v1-49.

2. Louis DN, Ohgaki H, Wiestler OD, Cavenee WK, Burger PC, Jouvet A, Scheithauer BW, Kleihues P. The 2007 WHO classification of tumours of the central nervous system. Acta Neuropathol. 2007; 114:97-109.

3. Ohgaki H, Kleihues P. Epidemiology and etiology of gliomas. Acta Neuropathol. 2005; 109:93-108.

4. Andersson U, Guo D, Malmer B, Bergenheim AT, Brännström T, Hedman H, Henriksson R. Epidermal growth factor receptor family (EGFR, ErbB2-4) in gliomas and meningiomas. Acta Neuropathol. 2004; 108:135-42.

5. Wrensch M, Wiencke JK, Wiemels J, Miike R, Patoka J, Moghadassi M, McMillan A, Kelsey KT, Aldape K, Lamborn KR, Parsa AT, Sison JD, Prados MD. Serum IgE, tumor epidermal growth factor receptor expression, and inherited polymorphisms associated with glioma survival. Cancer Res. 2006; 66:4531-41.

6. Ohgaki H, Dessen P, Jourde B, Horstmann S, Nishikawa T, Di Patre PL, Burkhard C, Schüler D, Probst-Hensch NM, Maiorka PC, Baeza N, Pisani P, Yonekawa Y, et al. Genetic pathways to glioblastoma: a population-based study. Cancer Res. 2004; 64:6892-99.

7. Ohgaki H, Kleihues P. Genetic alterations and signaling pathways in the evolution of gliomas. Cancer Sci. 2009; 100:2235-41.

8. Ohgaki H, Kleihues P. Population-based studies on incidence, survival rates, and genetic alterations in astrocytic and oligodendroglial gliomas. J Neuropathol Exp Neurol. 2005; 64:479-89.

9. Hou WG, Ai WB, Bai XG, Dong HL, Li Z, Zhang YQ, Xiong LZ. Genetic variation in the EGFR gene and the risk of glioma in a Chinese Han population. PLoS One. 2012; 7:e37531.

10. Liu HB, Peng YP, Dou CW, Su XL, Gao NK, Tian FM, Bai J. Comprehensive study on associations between nine SNPs and glioma risk. Asian Pac J Cancer Prev. 2012; 13:490508 .

11. Wang X, Zhang H, Wang D, Li X. Association of genetic polymorphisms of EGFR with glioma in a Chinese population. Genet Test Mol Biomarkers. 2015; 19:59-62.

12. Nagane M, Coufal F, Lin H, Bögler O, Cavenee WK, Huang HJ. A common mutant epidermal growth factor receptor confers enhanced tumorigenicity on human glioblastoma cells by increasing proliferation and reducing apoptosis. Cancer Res. 1996; 56:5079-86.

13. Connor AE, Baumgartner RN, Baumgartner KB, Pinkston CM, John EM, Torres-Mejía G, Hines LM, Giuliano AR, Wolff RK, Slattery ML. Epidermal growth factor receptor (EGFR) polymorphisms and breast cancer among Hispanic and non-Hispanic white women: the Breast Cancer Health Disparities Study. Int J Mol Epidemiol Genet. 2013; 4:23549.

14. Sasaki H, Okuda K, Shimizu S, Takada M, Kawahara M, Kitahara N, Okumura M, Matsumura A, Iuchi K, Kawaguchi T, Kubo A, Kawano O, Yukiue H, et al. EGFR $\mathrm{R} 497 \mathrm{~K}$ polymorphism is a favorable prognostic factor for advanced lung cancer. J Cancer Res Clin Oncol. 2009; 135:313-18.

15. Perez CA, Chen H, Shyr Y, Courtney R, Zheng W, Cai Q, Hwang M, Jaboin J, Schleicher S, Moretti L, Wills M, Smith JA, Lu B. The EGFR polymorphism rs884419 is associated with freedom from recurrence in patients with resected prostate cancer. J Urol. 2010; 183:2062-69.

16. Andersson U, Schwartzbaum J, Wiklund F, Sjöström S, Liu Y, Tsavachidis S, Ahlbom A, Auvinen A, Collatz-Laier H, Feychting M, Johansen C, Kiuru A, Lönn S, et al. A comprehensive study of the association between the EGFR and ERBB2 genes and glioma risk. Acta Oncol. 2010; 49:767-75.

17. Gabriel S, Ziaugra L, Tabbaa D. SNP genotyping using the Sequenom MassARRAY iPLEX platform. Current protocols in human genetics. 2009. doi: 10.1002/0471142905.hg0212s60.

18. Thomas RK, Baker AC, Debiasi RM, Winckler W, Laframboise T, Lin WM, Wang M, Feng W, Zander T, MacConaill L, Lee JC, Nicoletti R, Hatton C, et al. Highthroughput oncogene mutation profiling in human cancer. Nat Genet. 2007; 39:347-51.

19. Bland JM, Altman DG. Statistics notes. The odds ratio. BMJ. 2000; 320:1468.

20. Solé X, Guinó E, Valls J, Iniesta R, Moreno V. SNPStats: a web tool for the analysis of association studies. Bioinformatics. 2006; 22:1928-29.

21. Shi YY, He L. SHEsis, a powerful software platform for analyses of linkage disequilibrium, haplotype construction, and genetic association at polymorphism loci. Cell Res. 2005; 15:97-98.

22. Hawley ME, Kidd KK. HAPLO: a program using the EM algorithm to estimate the frequencies of multi-site haplotypes. J Hered. 1995; 86:409-11. 